\title{
Immunodiagnostic Potential of an In-Vitro Interferon-Gamma Release Assay for Latent Tuberculosis Infection Among Apparently Healthy Individuals in Okada Community, Nigeria
}

\author{
Benson Olu Akinshipe ${ }^{1, *}$, Peter Chinedu Ezeani², Kester Awharentomah Digban ${ }^{3}$, \\ Friday Alfred Ehiaghe ${ }^{4}$, Emmanuel Babatunde Adedeji ${ }^{5}$, Joy Imuetinyan Ehiaghe ${ }^{6}$ \\ ${ }^{1}$ Departments of Medical Microbiology, School of Clinical Medicine, College of Health Sciences, Igbinedion University \& Igbinedion \\ University Teaching Hospital, Okada, Nigeria \\ ${ }^{2}$ Kamorass Specialist Clinics Laboratories, Victoria Island, Lagos, Nigeria \\ ${ }^{3}$ Department of Medical Laboratory Science, College of Health Sciences, Igbinedion University, Okada, Nigeria \\ ${ }^{4}$ Department of Hematology, College of Health Sciences, Igbinedion University, Okada, Nigeria \\ ${ }^{5}$ Environmental Biology Research Unit, University Of Ibadan, Ibadan, Nigeria \\ ${ }^{6}$ Lahor Research Laboratory and Medical Centre, Benin City, Nigeria
}

Email address:

bensonakinshipedr01@yahoo.com (B. O. Akinshipe)

${ }^{*}$ Corresponding author

To cite this article:

Benson Olu Akinshipe, Peter Chinedu Ezeani, Kester Awharentomah Digban, Friday Alfred Ehiaghe, Emmanuel Babatunde Adedeji, Joy Imuetinyan Ehiaghe. Immunodiagnostic Potential of an In-Vitro Interferon-Gamma Release Assay for Latent Tuberculosis Infection Among Apparently Healthy Individuals in Okada Community, Nigeria. International Journal of Immunology. Vol. 5, No. 3, 2017, pp. 41-48. doi: $10.11648 /$ j.iji.20170503.11

Received: March 4, 2017; Accepted: March 24, 2017; Published: May 9, 2017

\begin{abstract}
A major challenge in the global Tuberculosis (TB) control is the diagnosis and treatment of Latent Tuberculosis Infection (LTBI). In the absence of any reference standard test for the diagnosis of LTBI, this study set out to compare the performance of the two current immune-based tests, Tuberculin Skin Test (TST) and Quantiferon-TB Gold In-Tube (QFTGIT) ELISA in the diagnosis of LTBI. Two sets of diagnostic results for 196 apparently healthy volunteers from a cross-section of Okada Community, Edo State, Nigeria were compared in terms of age, occupation, BCG-vaccination status, prior TST and cigarette smoking history. Overall, $56(28.6 \%)$ and $81(41.3 \%)$ of the subjects were diagnosed with LTBI by the QFT-GIT test and TST respectively. The LTBI prevalence as assessed by the QFT-GIT test was significantly higher among the non-BCGvaccinated, compared to the $B C G$-vaccinees $\left(X^{2}=18.79, \mathrm{df}=1, \mathrm{p}=0.0001\right)$. The highest concordance $(\mathrm{QFT}-\mathrm{GIT}+\mathrm{ve} / \mathrm{TST}+\mathrm{ve})$ was found in the occupation categories $(\mathrm{R}=-0.009, \mathrm{p}=0.747)$ and the highest discordance $(\mathrm{QFT}-\mathrm{GIT}-\mathrm{ve} / \mathrm{TST}+\mathrm{ve})$ was with respect to the $\mathrm{BCG}$-vaccination status $(\mathrm{R}=-0.194, \mathrm{p}=0.046)$. The disparity in the performance of the two tests is attributable to the high false - positive TST results, which is a direct reflection of high $(90.8 \%)$ BCG vaccination level among the study population. It is advocated that the two-step testing approach, using the QFT-GIT assay as a confirmatory test for LTBI after initial positive screening by the TST, be introduced into the TB control strategy in TB - laden communities with high BCG vaccination coverage.
\end{abstract}

Keywords: Interferon-gamma Release Assay, Tuberculin Skin Test, Latent Tuberculosis Infection, Immunodiagnostic, Okada 


\section{Introduction}

Tuberculosis (TB) continues to be one of the leading infectious causes of morbidity and mortality and remains a major public health problem worldwide, despite progress being made globally to prevent and control the disease. The huge majority of TB cases and TB deaths occur in the developing countries. Nigeria, the most populous country in Africa, leads the rest of the continent in the number of TB cases, and is currently rated 10th globally among the 22 High TB-burden Countries (HBCs) [1]. Although the expansion of Direct Observation Therapy Short-course (DOTS) and the Stop TB Partnership strategy have saved millions of lives in Nigeria, however, the incidence of TB is not declining as rapidly as expected in the low-income country [2].This is due in part to the emergence of multidrug - resistant TB (MTB), increasing prevalence of HIV/AIDS among TB patients[3], and also because of apparent deficiencies in the existing TB diagnostics pipeline in Nigeria.

TB disease is the result of a complex interaction between the causative organism, mycobacterium tuberculosis (MTb), and the host's innate and adaptive immune responses. In the vast majority of the infected individuals, an effective cellmediated immune response shortly after infection results in asymptomatic infection, which may remain undiagnosed and could reactivate later in life to cause active TB disease [4], [5]. The identification and treatment of this huge reservoir termed Latent Tuberculosis Infection (LTBI), is a key element in the post-2015 strategy for global TB control [1]. While most high income countries have incorporated effective LTBI control strategy into their TB control programme and elimination campaigns, this measure is still limited or virtually non-existent in most resource-limited countries [1].Tuberculosis control programmes in developing countries are greatly haunted by low detection rates and LTBIs that constitute hidden pools that continuously feed new cases. Accurate diagnosis of LTBI and appropriate treatment of probable cases - to- be reduce the risk of progression to overt disease and increase case detection rates [6].The Nigeria TB control programme has found it necessary to couple diagnosis and treatment of active TB disease with new approaches to detect LTBI [3].

Making a diagnosis of LTBI is constrained by a low bacteria load that makes it impossible to directly detect $\mathrm{MTb}$ and a weak humoral immune response that makes serological testing unreliable [7]. Therefore, the diagnostic options for LTBI are cell-mediated immune- based, given that none of the three options (i.e, smears, culture, nucleic acid amplification tests) for active TB work for LTBI [8].

There are currently two cell-mediated immune-based test principles for diagnosis of LTBI, the 'in-vivo' tuberculin skin test (TST) and the 'in-vitro' interferon gamma release assays (IGRAs). Until recently, the TST was the only tool available for diagnosing LTBI in Nigeria. However, the TST is known to have several biologic, operational and interpretational drawbacks that may be overcome by using the IGRAs, which have been adopted for diagnosing LTBI in many industrialized countries [9].The latest commercial IGRA, the whole-blood Quantiferon-TB Gold In-Tube (QFT-GIT) test is based on the observation that the CD4+ T lymphocytes of an individual who acquired $\mathrm{MTb}$ infection will respond by secreting the inflammatory cytokine interferon- gamma (IFN$\gamma$ ), when re-stimulated with a cocktail of highly M.Tbspecific antigens: Early Secreted Antigenic Target-6 (ESAT6), Culture Filtrate Protein-10 (CFP-10), and Tuberculosis 7.7 antigen (TB.7.7)(P4)), which are absent from most nontuberculous mycobacteria (NTM) and Bacille-Calmette Guerin (BCG) vaccine [10]. Interferon-gamma release assays have been found to be affected by factors like ethnic background, immune status of the population and highversus-low TB incidence settings[11], [12].The QFT-GIT and the TST tests have been widely evaluated in active TB patients in high-income, low TB-incidence countries [13], and the information on the varied levels of "sensitivities" and "specificities" of the two immune-based tests for LTBI that have been reported in these countries are inappropriately extrapolated to low and middle-income settings with high background TB infection rates.

Up to date, most epidemiologic data on LTBI is from the industrialized nations. So far, only two relevant systematic investigations using the QFT-GIT In-Tube test (in the diagnosis of active pulmonary TB) have been reported from Nigeria [14], [15]. To our informed knowledge, no work has been published from Nigeria using the QFT-GIT test for the rapid detection of the huge reservoir of LTBI which, if undiagnosed and untreated, constitutes a continuous source of TB transmission in the already TB-burdened country. Thus, further assessment is needed on the performance of the QFT-GIT test from Nigeria, where it is relatively new.

As part of our TB infection control program, this study set out to investigate the performance of the new ELISA-based, whole- blood QFT-GIT for LTBI diagnosis, prior to its implementation in our setting. We screened for LTBI in a mixed- age population of asymptomatic, apparently healthy individuals in our community by the dual testing approach, using simultaneously the TST and QFT-GIT immunodiagnostic tests. The study involved a head-to-head comparison of the two tests in the hope of improving the diagnosis of new LTBIs that can be targeted for preventive therapy in our high TB-endemic community.

\section{Study Population and Setting}

Three criteria were used in selecting the 'healthy' individuals in this study. These were that (1) the subject must not be bacteriological- culture confirmed TB patient on antiTB treatment, (2) the subject must not be immunosuppressed person (such as HIV/AIDs, diabetes mellitus patient on immuno-suppressant drug(s) or pregnant woman), and (3) must have attained the age of 6years as at the time of this study.

From March 2016 to July 2016, two hundred (200) individuals who fulfilled the three stated criteria were identified and registered during routine medical check-up at 
the outpatient department (OPD) of Igbinedion University Teaching Hospital (IUTH), Okada, Edo State, Nigeria. It has an estimated population of 29,000 inhabitants based on the 2005 Nigeria's National Population Census. A large number of the people are students, staff of the organized private and public sectors, or engage in one form of occupation or the other--all centered around the educational and training background of the area. Written Informed consent was given by each adult participant or from parent(s) or guardian(s) of children. Ethical clearance was obtained before the commencement of this study from the IUTH Research Ethics Committee and permission was given by the management of the Kamorass Specialist Clinics Laboratories, Victoria Island, Lagos, Nigeria for the use of their Immuno-diagnostic laboratory facilities.

\subsection{Questionnaire}

After enrollment, each of the 200 study volunteers filled a semi-structured questionnaire administered on initial encounter during routine medical check-up using pen and paper format. The questionnaire covered socio-demographic data (age, gender, marital status, ethnicity, level of education, occupation and workplace), risk factors for TB such as cigarette smoking, BCG vaccination, prior TST and chest radiographic findings. BCG vaccination status was verified by scar(s) or vaccination records.

Information supplied were confirmed from subject medical records with the IUTH or at the Okada Health Centre and seen to fulfill the healthy population selection criteria, before participation in this study

\subsection{Quantiferon-TB Gold In-Tube (QFT-GIT) Test}

All the 200 enrolled participants were subjected to the Quantiferon-TB Gold-In-Tube (QFT-GIT) test (Cellestis Limited, Carnegie, Australia), which was performed in two stages according to the manufacturer's instructions. First $3.0 \mathrm{ml}$ of venepuncture-collected whole-blood was drawn into heparinized tube and $1.0 \mathrm{ml}$ each was incubated with aliquots of antigen-free Negative control, MTb-specific antigens (ESAT-6, CFP-10, TB7.7 (P4)), or Tcell-mitogen (phytohemagglutinin) Positive control for $16-24$ hours at $37^{\circ} \mathrm{C}$ in a carbon dioxide incubator. During the overnight incubation, the reaction tubes were transported to the Kamorass Specialist Clinics Laboratories, Lagos for further processing by one of us trained as medical laboratory scientist (Immunology). Thus, the plasma from each tube was harvested by centrifugation and tested in batches of 40 samples per assay for the presence of Interferon gamma by enzyme-linked immunosorbent assay (ELISA).

In brief, a $50 \mu 1$ each of the reconstituted Kit standard (lyophilized human IFN- $\gamma$ ), the plasma sample, the Nil and Positive mitogen controls were dispensed into appropriatelylabelled plastic wells. A $50 \mu 1$ of the working conjugate reagent (monoclonal antibody conjugated to IFN- $\gamma$ ) was dispensed into each well and mixed thoroughly for 1 minute at $600 \mathrm{rpm}$ with the aid of a microplate shaker. The resultant mixture was incubated at $25^{\circ} \mathrm{C}$ in the dark for 2 hours. The solution was discarded and the microplate wells washed three times with diluted wash buffer using the automatic ELISA washer (Bio-Tek Instruments Inc, USA). $100 \mu \mathrm{L}$ of tetramethylbenzidine one-step Enzyme substrate solution was added to each microplate well and mixed thoroughly before incubation at $25^{\circ} \mathrm{C}$ in the dark for 30 minutes. $100 \mu$ of the kit Enzyme Stopping- solution was added to each of the wells in the same order as the substrate. The optical density (OD) of the color developed in each well was measured with the aid of the ELISA reader (Bio-Tek Instruments Inc, USA) at $450 \mathrm{~nm}$ wavelength within 1 hour of stopping the reaction. The calculation of the results was based on the QFT-GIT Analysis 2.23 software programme provided by the Kit manufacturer. The amount of IFN- $\gamma$ released was determined by subtracting the amount in the Nil from the amount in the MTb (ESAT-6-, CFP-10-, TB7.7 (P4)) - or mitogenstimulated plasma.

Positive response value of $\geq 0.35 \mathrm{IU} / \mathrm{ml}$ of IFN-gamma was used as the cut-off, Negative control concentration of at least $8.0 \mathrm{IU}$ per $\mathrm{ml}$ and positive difference value of less than $0.5 \mathrm{IU} / \mathrm{ml}$ was considered "indeterminate"(based on the manufacturer's guidelines).Volunteers with indeterminate QFT-GIT test results were excluded from the study population because the test could not be repeated since test had deployed.

The investigator who performed the IFN-y ELISA assay was blinded to the identity of the coded sample.

\subsection{Tuberculin Skin Test (TST)}

The enrolled subjects were skin-tested using a modified Mantoux method performed by one of us (Microbiologist) who was not involved in patient recruitment and bio- data collection. Briefly, immediately following blood drawn from the right hand for the QFT-GIT test, $0.1 \mathrm{ml}$ dose of 5-TU (Tuberculin Unit) of purified protein derivative (PPD) RT23 (Statens serum Institute, Copenhagen, Denmark)was injected intradermally into the volar surface of the forearm. Correct injection was indicated by the production of palpable elevation of the skin (weal) $6-10 \mu \mathrm{m}$ in horizontal transverse diameter. The skin test was read 48-72hours later (after injection) using flexible plastic ruler. Interpretation of the results was done according to American Thoracic Society Guidelines (16), presence of palpable indurations of $\geq 10 \mathrm{~mm}$ was regarded as positive TST. A positive TST indicates that the reacting person has at some point in time become infected with a mycobacterium that has left an immunological imprint [17].

\subsection{Statistical Analysis}

Demographic, clinical and laboratory raw data from each study participant were collated with the aid of a Microsoft access Database (Microsoft Corp., Redmond, Australia). Statistical analysis was performed using the Statistical Package for Social Sciences (SPSS) (Version 20.0; SPSS Inc., Chicago, IL, USA) for windows. The calculation of the 
results of IFN-gamma released was based on the QFT-GIT analysis 2.23 software programme provided by the manufacturer (Cellestis Ltd, Carnegie, Australia). Chisquare $\left(\mathrm{X}^{2}\right)$, Fisher's exact test, Mcnemar and Mann-Whitney tests were used for testing associations between TST and QFT-GIT tests. Results were presented as frequencies and percentages. Agreement between test results was analyzed by using Kappa (k) coefficients. The significance level was set at $\mathrm{P} \leq 0.05$ in all analysis.

\subsection{Limitation of Study}

The limitation of this study is the lack of a reference gold standard test for the diagnosis of LTBI.

Table 1. Basic Characteristics of the Study Participants $(N=196)$.

\begin{tabular}{ll}
\hline Characteristics & n (\%) \\
\hline Sex & \\
Male & $101(51.5)$ \\
Female & $95(48.5)$ \\
Age group (Years) & \\
$6-15$ & $34(17.3)$ \\
$16-25$ & $38(19.4)$ \\
$26-35$ & $35(17.9)$ \\
$36-45$ & $30(15.3)$ \\
$46-55$ & $28(14.3)$ \\
$56-65$ & $31(15.8)$ \\
Occupation & \\
Schooling & $42(21.4)$ \\
Health care & $36(18.4)$ \\
Trading & $27(13.8)$ \\
Farming & $29(14.8)$ \\
Artisan & $26(13.3)$ \\
Other & $25(12.8)$ \\
Unemployed & $11(5.6)$ \\
Smoking Status & \\
Never Smoked & $162(82.7)$ \\
Current Smoker & $21(10.7)$ \\
Previous Smoker & $13(6.6)$ \\
BCG Vaccination & \\
Yes & $178(90.8)$ \\
No & $18(9.2)$ \\
\hline
\end{tabular}

Table 2. Results of LTBI Prevalence According to Age Group as Assessed by QFT-GIT/TST.

\begin{tabular}{|c|c|c|c|c|c|c|c|}
\hline \multirow{2}{*}{$\begin{array}{l}\text { Age Group } \\
\text { (Years) }\end{array}$} & \multirow{2}{*}{$\mathbf{N}$} & \multicolumn{2}{|l|}{ QFT-GIT } & \multirow{2}{*}{$\begin{array}{l}\text { Test Statistics } \\
\text { (p value) }\end{array}$} & \multicolumn{2}{|l|}{ TST } & \multirow{2}{*}{$\begin{array}{l}\text { Test Statistics } \\
\text { (p value) }\end{array}$} \\
\hline & & Positive (\%) & Negative (\%) & & Positive (\%) & Negative (\%) & \\
\hline $6-15$ & 34 & $4(11.8)$ & $30(88.2)$ & \multirow{7}{*}{$\begin{array}{l}X^{2}=11.4 \\
\mathrm{P}=0.045\end{array}$} & $7(20.6)$ & $27(79.4)$ & \multirow{7}{*}{$\begin{array}{l}X^{2}=12.6 \\
\mathrm{P}=0.028\end{array}$} \\
\hline $16-25$ & 38 & $6(15.8)$ & $32(84.2)$ & & $13(34.2)$ & $25(65.8)$ & \\
\hline $26-35$ & 35 & $14(40.0)$ & $21(60.0)$ & & $19(54.3)$ & $16(45.7)$ & \\
\hline $36-45$ & 30 & $13(43.3)$ & $17(56.7)$ & & $17(56.7)$ & $13(43.3)$ & \\
\hline $46-55$ & 28 & $9(32.1)$ & $19(67.9)$ & & $13(46.4)$ & $15(53.6)$ & \\
\hline $56-65$ & 31 & $10(32.3)$ & $21(67.7)$ & & $12(38.7)$ & $19(61.3)$ & \\
\hline Total & 196 & $56(28.6)$ & $140(71.4)$ & & $81(41.3)$ & $115(58.7)$ & \\
\hline
\end{tabular}

Table 3. Prevalence of LTBI in different Occupations as Assessed by QFT-GIT/ TST.

\begin{tabular}{|c|c|c|c|c|c|c|c|}
\hline \multirow{2}{*}{ Occupation } & \multirow{2}{*}{$\mathbf{N}$} & \multicolumn{2}{|l|}{ QFT-GIT } & \multirow{2}{*}{$\begin{array}{l}\text { Test Statistics } \\
\text { (p value) }\end{array}$} & \multicolumn{2}{|l|}{ TST } & \multirow{2}{*}{$\begin{array}{l}\text { Test Statistic } \\
\text { (p value) }\end{array}$} \\
\hline & & Positive (\%) & Negative (\%) & & Positive (\%) & Negative (\%) & \\
\hline Schooling & 42 & $16(38.1)$ & $26(61.9)$ & \multirow{8}{*}{$\begin{array}{l}X^{2}=39.8 \\
\mathrm{P}=0.02\end{array}$} & $12(28.6)$ & $30(71.4)$ & \multirow{8}{*}{$\begin{array}{l}X^{2}=39.8 \\
\mathrm{P}=0.02\end{array}$} \\
\hline Health care & 36 & $17(47.2)$ & $19(52.8)$ & & $26(72.2)$ & $10(27.8)$ & \\
\hline Trading & 27 & $9(33.3)$ & $18(66.7)$ & & $7(25.9)$ & $20(74.1)$ & \\
\hline Farming & 29 & $3(10.3)$ & $26(89.7)$ & & $11(37.9)$ & $18(62.1)$ & \\
\hline Artisan & 26 & $7(26.9)$ & $19(73.1)$ & & $10(38.5)$ & $16(61.5)$ & \\
\hline Other & 25 & $2(8.0)$ & $23(92.0)$ & & $9(36.0)$ & $16(64.0)$ & \\
\hline Unemployed & 11 & $2(18.2)$ & $9(81.8)$ & & $6(54.5)$ & $5(45.5)$ & \\
\hline Total & 196 & $56(28.6)$ & $140(71.4)$ & & $81(41.3)$ & 115 (58.7) & \\
\hline
\end{tabular}


Table 4. Prevalence of LTBI According to Smoking Status as Assessed by QFT-GIT/TST.

\begin{tabular}{|c|c|c|c|c|c|c|c|}
\hline \multirow{2}{*}{ Smoking status } & \multirow{2}{*}{$\mathbf{N}$} & \multicolumn{2}{|l|}{ QFT-GIT } & \multirow{2}{*}{$\begin{array}{l}\text { Test Statistics } \\
\text { (p value) }\end{array}$} & \multicolumn{2}{|l|}{ TST } & \multirow{2}{*}{$\begin{array}{l}\text { Test Statistics } \\
\text { (p value) }\end{array}$} \\
\hline & & Positive (\%) & Negative (\%) & & Positive (\%) & Negative (\%) & \\
\hline Never smoked & 162 & $25(15.4)$ & $137(84.6)$ & \multirow{4}{*}{$\mathrm{P}=<0.003$} & $61(37.7)$ & $101(62.3)$ & \multirow{4}{*}{$\mathrm{P}=<0.005$} \\
\hline Current smoker & 21 & $19(90.5)$ & $2(9.5)$ & & $15(71.4)$ & $6(28.6)$ & \\
\hline Previous Smoker & 13 & $12(92.3)$ & $1(7.7)$ & & $5(38.5)$ & $8(61.5)$ & \\
\hline Total & 196 & $56(28.6)$ & $140(71.4)$ & & $81(41.3)$ & $115(58.7)$ & \\
\hline
\end{tabular}

Table 5. Assessment of LTBI Prevalence by QFT-GIT/TST according to BCG Vaccination Status.

\begin{tabular}{llllllll}
\hline & & QFT-GIT & & Test Statistics & TST & & Test Statistics \\
\hline BCG Vaccination & N(\%) & Positive n (\%) & Negative n (\%) & & Positive n (\%) & Negative n (\%) & \\
\hline Yes & $178(90.8)$ & $43(24.2)$ & $135(75.8)$ & X2 $=18.79$ & $72(40.4)$ & $106(59.6)$ & X2 $=1.64$ \\
No & $18(9.2)$ & $13(72.2)$ & $5(27.8)$ & df 1 & $9(50.0)$ & $9(50.0)$ & df $=1$ \\
TOTAL & 196 & 56 & 140 & $\mathrm{p}=0.0001$ & 81 & 115 & $\mathrm{p}=0.20$ \\
\hline
\end{tabular}

Table 6. Test of agreement between QFT-GIT and TST Positivity for LTBI among the participants.

\begin{tabular}{|c|c|c|c|c|c|c|}
\hline Characteristic & QFT-GITPositive & TSTPositive & kappa & P value & Mann-Whitney U & P value \\
\hline \multicolumn{7}{|l|}{ Age group (Years) } \\
\hline $6-15$ & $4(11.8)$ & 7 (20.6) & \multirow{6}{*}{-0.018} & \multirow{6}{*}{0.393} & \multirow{6}{*}{2098} & \multirow{6}{*}{0.448} \\
\hline $16-25$ & $6(15.8)$ & $13(34.2)$ & & & & \\
\hline $26-35$ & $14(40.0)$ & $19(54.3)$ & & & & \\
\hline $36-45$ & $13(43.3)$ & $17(56.7)$ & & & & \\
\hline $46-55$ & $9(32.1)$ & $13(46.4)$ & & & & \\
\hline $56-65$ & $10(32.3)$ & $12(38.7)$ & & & & \\
\hline \multicolumn{7}{|l|}{ Occupation } \\
\hline Schooling & $16(38.1)$ & $12(28.6)$ & \multirow{7}{*}{-0.009} & \multirow{7}{*}{0.747} & \multirow{7}{*}{1770} & \multirow{7}{*}{$0.026^{*}$} \\
\hline Health care & $17(47.2)$ & $26(72.2)$ & & & & \\
\hline Trading & $9(33.3)$ & $7(25.9)$ & & & & \\
\hline Farming & $3(10.3)$ & $11(37.9)$ & & & & \\
\hline Artisan & $7(26.9)$ & $10(38.5)$ & & & & \\
\hline Other & $2(8.0)$ & $9(36.0)$ & & & & \\
\hline Unemployed & $2(18.2)$ & $6(54.5)$ & & & & \\
\hline \multicolumn{7}{|l|}{ Smoking status } \\
\hline Never smoked & $25(15.4)$ & $61(37.7)$ & \multirow{3}{*}{-0.107} & \multirow{3}{*}{$0.005^{*}$} & \multirow{3}{*}{1530} & \multirow{3}{*}{$0.005^{*}$} \\
\hline Current smoker & $19(90.5)$ & $15(71.4)$ & & & & \\
\hline Previous SmokerBCG vaccination & $12(92.3)$ & $5(38.5)$ & & & & \\
\hline Yes & $43(24.2)$ & $72(40.4)$ & \multirow{2}{*}{-0.194} & \multirow[t]{2}{*}{0.046} & \multirow[t]{2}{*}{1430} & \multirow[t]{2}{*}{0.004} \\
\hline No & $13(72.2)$ & $9(50.0)$ & & & & \\
\hline
\end{tabular}

$* \mathrm{P}<0.05$ is considered significant

\section{Results}

In all, 200 apparently healthy individuals who fulfilled the selection criteria were recruited for this study. Four of the subjects were excluded from the final results: one male subject aged 16 years did not return for the reading of the TST result while three subjects (one female and two males) in the 56-65 years age group were excluded because of indeterminate QFT - GIT test results. A final study population size of 196 was achieved.

Participant Demographic Characteristics

Table 1 shows the basic characteristics of the 196 study participants with complete set of immuno- diagnostic results for LTBI. Nearly equal number of male 101 (51.5\%) and female $95(48.5 \%)$ were tested by the QFT-GIT and TST. The age of the subjects ranged from $6-65$ years, the age group 16-25 years had the highest frequency of 38(19.4\%). Among the participants, $42(21.4 \%)$ were schooling, 36 (18.4\%) health care workers, 29(14.8\%) farmers, $27(13.8 \%)$ traders, 26(13.3\%) artisanal workers and $25(12.8 \%)$ were engaged in a variety of other jobs; whereas 11(5.6\%) reported to be unemployed as at the time of this study. A majority $178(90.5 \%)$ of the subjects had received $\mathrm{BCG}$ vaccination as evidenced from scar(s) or vaccination records. Of these participants, $162(82.7 \%)$ reported to have never smoked, 21(10.7\%) as current smokers while $13(6.6 \%)$ were ex-smokers

Assessment of LTBI by QFT - GIT and TST

The results as shown in Table 2 revealed an overall LTBI prevalence of $56(28.6 \%)$ as assessed by QFT - GIT assay or $81(41.3 \%)$ by TST among the 196 participants tested. Significant increase in the LTBI prevalence rate as detected by the QFT -GIT test was observed in the $26-35$ years and $36-45$ years age groups, which recorded $40.0 \%$ and $43.3 \%$ positive rates respectively $\left(\mathrm{X}^{2}=11.4, \mathrm{p}=0.045\right)$, while the corresponding rates as detected by the TST in these age groups were also significantly increased at $54.3 \%$ and $56.7 \%$ respectively when compared to the other age groups $\left(\mathrm{X}^{2}=\right.$ 12.6, $\mathrm{p}=0.028)$. As shown in Table 3, the QFT-GIT test and TST positive rates for LTBI were significantly higher, $47.2 \%$ and $72.2 \%$ respectively, among the health care workers compared to the other job categories. 
The prevalence of LTBI as determined by QFT-GIT test was significantly higher in both current smokers $19(90.5 \%)$ and previous smokers $12(92.3 \%)$, compared to $25(15.4 \%)$ rate among the never smoked category; whereas the corresponding LTBI prevalence rates as assessed by the TST for current smokers,previous smokers and non-smokers were $71.4 \%, 38.5 \%$ and $37.7 \%$ respectively (Table 4). The assessment of LTBI prevalence according to BCGvaccination status is presented in Table 5. The rate of detection by QFT-GIT test is significantly higher $13(72.2 \%)$ in non-BCG- vaccinated subjects compared to the BCGvaccinated individuals with $43(24.2 \%)$ positive immune response $\operatorname{rate}\left(\mathrm{x}^{2}=18.79\right.$, df $\left.1, \mathrm{p}=0.0001\right)$.Conversely, there was no statistically significant difference in the LTBI prevalence rate between the BCG-vaccinated 72 (40.4\%) and non-BCG-vaccinated participants $9(50.0 \%)$ as detected by TST. $\left(X^{2}=16.4\right.$, df $\left.1, p=0.20\right)$. LTBI

Test of Agreement between QFT-GIT and TST tests for

Table 6 shows the correlation between the results of the QFT-GIT and TST tests for LTBI. The results among the different age groups and occupations gave poor insignificant agreement between the two tests $(\mathrm{R}=-0.018,-0.009 ; \mathrm{p}=$ $0.393,0.747)$ respectively. It was also observed that high level of QFT-GIT -ve/ TST +ve discordant results were observed among the health care workers. Smoking status shows significant poor agreement between the QFT-GIT and TST tests $(\mathrm{R}=-0.107, \mathrm{p}=0.005)$.However, significant increase in agreement was observed in the QFT-GIT and TST test results among current smokers $(\mathrm{U}=1530, \mathrm{p}=$ $0.005)$. There was very poor correlation between the two tests in the detection of LTBI among the BCG-vaccinated and non-BCG vaccinated individuals $(\mathrm{R}=-0.194, \mathrm{p}=0.046)$.

\section{Discussion}

The detection and treatment of Latent Tuberculosis Infection (LTBI) is an important strategy for TB control programme. Paradoxically, there are deficiencies in the existing TB diagnostics pipeline in the high TB-burden countries, such as Nigeria. Although the use of interferongamma release assays (IGRAs) for the detection of LTBI is wide- spread in developed countries [13], the tests are yet to be incorporated into the LTBI identification strategy in Nigeria. In the absence of a global reference standard test for the diagnosis of LTBI, we conducted a comparative study of the two current immune-based tests, the traditional TST and the latest IGRA (QFT-GIT test) for the detection of LTBI in Okada community, Edo State, Nigeria.

This report on a community-based cross-sectional survey of LTBI is one of the few so far recorded in Nigeria. Our results revealed that the sero-prevalence of LTBI in the Okada community ranged from $28.6 \%$ (as assessed by QFTGIT test) to $41.3 \%$ (as assessed by TST). The observed significantly higher overall LTBI prevalence rate of $81(41.3 \%)$ as detected by the TST compared to $56(28.6 \%)$ by QFT-GIT test in this study indicates that some participants who were diagnosed with LTBI based on TST results were found to be QFT-GIT negative(i.e TST +ve / QFT-GIT-ve). Similar variation in the performance of these two immunodiagnostic tests has been reported by other workers in developing countries [14], [19], [21].

The generally higher rate of LTBI recorded for TST compared to the QFT-GIT test, as confirmed in the present study, suggests that either TST could be more sensitive than the QFT-GIT in the detection of LTBI, or reflect a high rate of false-positive TST results in developing countries. This observation may be explained by exposure to nontuberculous bacteria in those developing countries, or the fact that the antigen used in TST (PPD) is a shared antigen present in M.tuberculosis and non-tuberculosis mycobacteria [22], but in QFT-GIT test, the antigens used (i.e, ESAT-6, CFP-10 and TB 7.7(P4)) are M tuberculosis-specific antigens and therefore considered to be more specific than TST in screening for LTBI [7]. The higher specificity of QFT-GIT for the diagnosis of LTBI is also supported by recent studies in developed countries, which indicate that Tuberculin Skin Testing has lower specificity as the test cannot differentiate between infection with MTb, prior vaccination with M.bovis BCG, or sensitization with environmental bacteria [9], [23], [24]. Conversely, unlike skin testing, the in-vitro QFT-GIT test might have been able to discriminate true-positive immune responses from anergy [25].

Taken together, the TST might have over-estimated the LTBI prevalence rate in this study. Therefore, the use of QFT-GIT test in LTBI surveillance would prevent some unnecessary prophylaxis and detect truly infected individuals more accurately, which will lead to more effective TB control in our community.

Our subjects with positive concordant results (i.e, QFTGIT +ve / TST +ve) were significantly older than those with negative concordant results (i.e, QFT-GIT -ve/TST -ve) which may indicate age-dependency of LTBI. This is to be expected as increasing age could be a risk for MTb infection because of longer time for potential exposure and because older persons might have been alive when TB was more prevalent in the environment. The association of older age with positive TST and QFT-GIT test results, as found in the present study, could also be attributed to MTb infection that accumulated over time in our community, but did not progress to active TB because of effective immune defense in our selected immuno-competent, healthy subjects.

The findings of this study showed that TST positive results for LTBI were highest among health-care workers $(72.2 \%)$, compared to a lower prevalence rate of $47.2 \%$ by QFT-GIT test recorded among this occupational group. The higher detection rate among health-care workers by TST was in agreement with Drobniewski et al [26] study, where the proportion of LTBI assessed by TST was highest among health-care professionals, compared to other work groups. This is possible because of the boosting effect of repeat TSTs during their employment [27], [19], to which health care workers tested in this study were similarly predisposed. In contrast, the QFT-GIT test which utilized M.Tb unique 
antigens (ESAT-6, CFP-10, TB.7.7 (P4)) is expected to be more specific for the detection of LTBI.

The high QFT-GIT positive rates of $90.5 \%$ and $92.3 \%$ among current- and ex - smokers respectively, and the corresponding high LTBI prevalence rate within the 26-35 years and 36-45 years age groups suggest that the test is well correlated with LTBI as smoking, which is commonly associated with these two youthful age groups, is a renowned risk factor for both LTBI and active TB [21],[28].

The observed significantly higher rate of LTBI detected by the QFT-GIT in the non-BCG- vaccinated subjects in this study, compared to their BCG- vaccinated counter-parts, indicates that the test is not affected by previous BCG vaccination as it utilized MTb specific antigens. However, the TST is known to show cross-reactivity with hypersensitivity in BCG-vaccinated and non-tuberculous mycobacterial (NTM) infections [29], therefore, lower specificity of TST is expected in the detection of LTBI in comparison with the QFT-GIT test; especially in population with high BCG-vaccination coverage such as is the case in the present study setting. The lower specificity of TST for LTBI in BCG-vaccinees has been previously reported by other workers [21].

Our results showed poor concordance between QFT-GIT and TST in the diagnosis of LTBI among the healthy individuals in the Okada community. Agreement among tests for LTBI varies widely in reported studies. This is to be expected as the TST, but not QFT-GIT test, is confounded by BCG vaccination (to which $90.8 \%$ of our study population have been exposed); and the variety of TB-burden settings [8]. Furthermore, agreement in these studies has been affected by interpretation criteria, co-existing diseases, HIVTB co-infection; and the differences in the biology and immunology of participants [10], [30].

The major operational constraint posed by the QFT-GIT test, as realized in the present work, is its high cost, which is substantially more than TST. It also requires equipment and consumables that translate into high costs for the health care delivery system. Combined together, these factors have limited the availability of the QFT-GIT test (for LTBI diagnosis) in the developing countries, such as Nigeria. However, it has been shown that the medical decrease in medical assessment (as occasioned by the QFT-GIT testing for LTBI in the present study), improves efficiency and costeffectiveness and fewer patients are inconvenienced by the long-term multi-drug TB treatment and its associated toxicities than if only TST was used [31]. Moreover, the invitro QFT-GIT test does not produce any boost effect or require a re-visit to the hospital for the reading of the result. Furthermore, as measurement of INF-gamma released in the QFT-GIT assay are carried out by machines, more objective, readout results are obtained more quickly than TST. Finally, as most TST results are recorded in individual patient records in developing countries, not in electronic databases, QFTGIT test results can be stored in laboratory databases for easy analysis and management in such settings.

\section{Conclusion}

This baseline study revealed that the prevalence of LTBI was high in the Okada community regardless of the immunodiagnostic method used. The TST gave higher overall LTBI prevalence rate compared to the QFT-GIT test. Given that there is no criterion reference standard test for the diagnosis of LTBI, it is hard to estimate the absolute sensitivities and specificities of the TST and QFT-GIT assays used in this study. Nevertheless, the ELISA - based QFTGIT test appears to be more specific for the detection of LTBI, and the pattern of discordance between these two immune-based tests, the QFT-GIT and TST, could be explained by the high proportion $(90.8 \%)$ of BCG vaccination coverage among our study population and the resultant high number of false - positive TST results. Therefore, the traditional TST technique is not reliable and adequate for the diagnosis of LTBI in our setting. It is suggested that the two-step TST and confirmatory QFT-GIT testing approach, as employed in the present work, would improve the effectiveness of LTBI identification strategy as an integral part of the TB control programme, particularly in our setting where data is currently not available on the performance of the QFT-GIT test, and generally in high TBendemic settings.

\section{References}

[1] World Health Organization (WHO) Tuberculosis Report (2016). http//www.who.int/mediacentre/factsheets/fs104/en /

[2] World Health Organization. Global tuberculosis control: epidemiology, strategy, financing: WHO report 2009. Geneva, Switzerland: World Health Organisation 2009. Available at http://www.int/tb/publications/global report/2009/pdf/report.

[3] Nigeria Tuberculosis Fact Sheet http://nigeria.usembassy.gov

(2012).

[4] Collins HL, Kaufmann SHE. Acquired immunity against bacteria. In: SHE Kaufmann, A Sher, R Ahmed (Eds.).Immunology of infectious Diseases. ASM Press, Washington DC 2002. Pp 207-221. Chapter 15

[5] Schluger N.W. The pathogenesis of tuberculosis: the first one hundred (and twenty- three) years. Am. J. Respir Cell Mol. Biol. 2005; 32: $251-256$

[6] Shakak AO, Khadil EAG, Musa AM, Salih KAM et al. Possible risk factors of progression to overt disease among individuals with latent tuberculosis infection in the Sudan. IJCR. 2013: 13: 1107-1110.

[7] PaiM, Zwerling A, Menzies D. Systematic review: T-cellbased assays for the diagnosis of latent tuberculosis infection: an update. Ann lntern Med 2008 ; 149: 177-184

[8] Lavlani A, Pareek M. A 100 year update on diagnosis of tuberculosis infection. Br.Med: Bull 2010; 93: 69-84.

[9] Pinto LM, Grenier J, Schumacher SG, Denkinger CM, Steingart KR, Pai M. Immunodiagnosis of Tuberculosis: State of the Art. Med. Princ. Pract. 2012;21:4-13 
[10] Babayigit C, Ozer B, Inandi T, Ozer C, Duran N, Gocmen O. Performance of QuantiFERON-TB Gold in -Tube test and Tuberculin Skin Test for diagnosis of latent tuberculosis infection in BCG vaccinated health care workers. Med Sci. Monit. 2014, 20: 521-529.

[11] Mazurek GH, Jereb J, Vernon A, Loblue P et al. CDC updated guidelines for using interferon gamma release assays to detect mycobacterium tuberculosis infection- United States, 2010. MMWR Recommd. Rep 2010; 59: 1-25.

[12] Lucas M, Nicol P, Mckinnon E, Whidborne R, Lucas A et al. A prospective large-scale study of methods for the detection of latent mycobacterium tuberculosis infection in refugee children. Thorax. 2010, 65(5) 442-448.

[13] Diel R, Goletti D, Ferrara G, Bothamley C et al. Interferon gamma release assays for the diagnosis of latent mycobacterium tuberculosis infection: a systematic review and meta-analysis. Eur Respir J. 2011;13: 88-99

[14] Adewole OO, Erhabor GE, Sogaolu MO, Onipede AO, Owiafe PK, Awopetu FO, Ota MO. Diagnostic utility of Quantiferon-TB Gold in-tube in active pulmonary Tuberculosis in Nigeria. West Afr J Med.2013, 32 (3): 180185.

[15] Nakaoka H, Lawson L, Bertel Squire S, Coulter B et al. Risk for Tuberculosis among children. Emerg Infect Dis 2006; 12 (9): $1383-1388$

[16] American Thoracic Society. Targeted tuberculosis testing and treatment of latent tuberculosis infection. Am J. RespirCrit Care Med 2000; 161: S221-S247.

[17] Reider HL, Chadha VK, Nagelkerke NJD, van Leth F, van der Werf M.J: Guidelines for conducting tuberculin skin test surveys in high prevalence countries. Int $\mathbf{J}$ Tuberc Lung Dis 2011; 15:S1-26.

[18] Adetifa IM, Ota MO, Jeffries DJ, Hammond A, Lugos M.D et al. Commercial interferon gamma release assays compared to the tuberculin skin test for diagnosis of mycobacteria tuberculosis infection in childhood contacts in the Gambia. Pediatr Infect. Dis J. 2010; 29 (5): 439.

[19] Kang YA, Lee HW, Yoon H I et al. Discrepancy between the tuberculin skin test and the whole-blood interferon-gamma assay for the diagnosis of latent tuberculosis infection in an intermediate tuberculosis-burden country. JAMA (2005), 293, 2756-2761.

[20] Legesse M, Ameni G, Mamo G, Medhin G et al. Performance of Quantiferon-TB Gold In-Tube (QFT-GIT) for the diagnosis of mycobacterium tuberculosis (MTb) infection in Afar Pastoralists, Ethiopia. BMC infectious Diseases, 2010, 10:354.
[21] Shanaube K, Hargreaves J, Fielding K, Schaap A et al. Risk factors Associated with Positive QuantiFERON-TB Gold InTube and Tuberculin Skin Tests Results in Zambia and South Africa. PLoS one, 2011, vol 6.No4, 18206.

[22] Farhat M, Greenaway C, Pai M, Menzies D. "False-positives tuberculinskin tests: what is the absolute effect of BCG and non-tuberculous mycobacteria?" International $\mathrm{J}$ of Tuberculosis and Lung Disease 2006, vol. 10 no 11, pp 11921204.

[23] Nienhaus A, Ringhausen PC, Costa J. T, Schabion A, Tripodi D. IFN- gamma release assay versus tuberculin skin test for monitoring TB infection in healthcare workers Expert Rev. Anti. Infect. Ther.2013; 11 (1), 37

[24] TrajmanA, Steffen RE, Menzies D. Interferon-Gamma Release Assays versus Tuberculin skin Testing for the diagnosis of Latent Tuberculosis infection: An overview of the evidence. Pulmonary Medicine, vol.2013 (2013)

[25] Choi JC, Shin JW, Kim JY et al. The effect of previous tuberculin skin test in the follow-up examination of wholeblood interferon-gamma assay in the screening of latent tuberculosis infection. Chest 2008; 133: 1415-1420.

[26] Drobniewski F, Balabanova Y, Zakamova E, Nilkolayevskyy V, Fedonn I (2007). Rates of latent tuberculosis in health care staff in Russia. PLoS Med.4 (2). 55.

[27] Menzies D "Interpretation of repeated tuberculin tests: boosting, conversion and reversion". Am J RespirCrit Care Med, vol. 159, no 1, pp. 15-21, 1999. View at Google scholar.

[28] Mirtskhulava V, Kampker R, Leonard MK, Tsertsvadze T, Diel R et al. Prevalence and risk factors for latent Tuberculosis infection among health care workers in Georgia. Int J. Tuberc Lung Disease 2008. 12; 513-519.

[29] Huebner RE, Schein MF, Bass JB Jr. The tuberculin skin test. Clin Infect Dis. 1993; 13: 968-975. doi 101093/clinds/17.6.968.

[30] Higuchi K, Kawabe Y, Mitarai S,Yoshiyama T, Harada N, Mori T. "Comparison of performance in two diagnostic methods for tuberculosis infection", Medical Microbiology and Immunology. 2009, vol. 198, no 1, pp 33-37.

[31] Rangaka MX, Wilkinson KA, Glynn JR, Ling D, Menzies D, Mwansa-Kambafwile, et al. "Predictive value of interferongamma release assays for incident active tuberculosis: a systematic review and meta-analysis", The Lancet Infectious Diseases 2012; vol 12. No. 1, pp 45-55.

[32] Quantiferon-TB Gold (in-tube method). Package insert. March 2013. Retrieved from Cellestis website. http://www. Cellestis. com/IRM/ company/show page. aspx (cpin-1171) 